\title{
Increased Shoulder Abduction Loads Decreases Volitional Finger Extension in Individuals with Chronic Stroke: Preliminary Findings
}

\author{
Yiyun Lan-IEEE Member, Jun Yao-EMBS Member, and Jules Dewald, IEEE Member
}

\begin{abstract}
The ability to open the paretic hand is greatly affected after a stroke. The loss of especially finger extension has been previously reported during isolated finger movements. However, activities of daily life require the combination of reaching and grasping which will require shoulder abduction. Shoulder abductor activity will result in concurrent elbow, wrist and finger flexion which is also referred to as the flexion synergy. Therefore as part of this study the effect of of shoulder abduction (SABD) loading on volitional finger extension in individuals with chronic stroke is investigated. We expect to observe that shoulder abduction loading will further decrease the already impaired volitional finger extension in individuals with chronic stroke.

A total of four moderately impaired individuals with chronic stroke and three age-matched able-bodied subjects participated in this study. Finger extension was recorded during hand open while subjects kept their arm extended at the end of a reach. The preliminary data showed that the maximal volitional finger extension was significantly decreased by increasing the SABD loads in individuals with chronic stroke, but not in age-matched able-bodied subjects.
\end{abstract}

\section{INTRODUCTION}

Stroke is the leading cause of adult disability in the United States [1]. The majority of stroke survivors report impaired upper extremity (UE) function, especially of the hand, is a major problem [2]. Due to the loss of hand function, $26 \%$ of stroke survivors are not able to live independently [3]. Most, if not all, previous studies of poststroke hand function have addressed the wrist and hand in isolation (i.e., with a static, fully supported arm). However, during activities of daily living, both functional arm and hand activity is necessary. Without a functional arm, the hand can only be used in a limited workspace. Without a functional hand, the arm will rarely be used. Both situations can lead to learned non-use of the paretic UE [4], constituting a major cause of chronic disability in this population. When a paretic arm and hand are used together, abnormal joint coupling (i.e., synergy) of the shoulder, elbow, wrist, and fingers may further increase hand dysfunction [5, 6]. This abnormal coupling is prevalent in most individuals with moderate to severe stroke. However, its impact on hand function has

Resrach supported by R01HD039343 research award from the NIH entitled "Effects of Neural Constraints on Movement in Stroke".

Yiyun Lan is currently a doctoral candidate in Interdepartmental Neuroscience Program at Northwestern University (NUIN), Chicago, IL, 60611, USA. (Email: yiyunlan2009@u.northwestern.edu).

Jun Yao is with Northwestern University, Chicago, IL 60611 USA. She is now a faculty member with the Department of Physical Therapy and Human Movement Science. (Email: j-yao4@northwestern.edu).

Jules Dewlad is currently the chair and professor of Department of Physical Therapy and Human Movement Science, Northwestern University, Chicago, IL. He is also a professor with the Biomedical Engineering Department. (Email: j-dewald@northwestern.edu). received very little attention. We currently know that when an individual with stroke abducts the arm against shoulder loads or reaches for a distant object, a significantly increased involuntary flexion force can be recorded at the wrist and fingers $[5,6]$. The key question - whether a voluntary drive of the hand can overcome this synergy induced involuntary flexion force-remains largely unanswered. The answer to this question will significantly impact whether individuals with stroke can regain control of the paretic hand while using the arm. So the objective of this study is to quantitatively describe the ability to open the hand as a function of SABD loading after a stroke.

\section{METHODS}

\section{A. Subjects}

Four individuals with moderate chronic stroke (mean FMA $=25 / 66$, Chedoke hand score $=4-5 / 7$, table 1 ) and three age-matched controls participated in this study. Individuals with stroke were recruited from the Clinical Neuroscience Research Registry, maintained by the Rehabilitation Institute of Chicago (RIC). All participants provided informed consent that was approved by investigational review board at Northwestern University.

\section{B. Experiment Setup and Protocol}

Isometric SABD force measurements were performed on the paretic arm of individuals with stroke and on the dominant arm of control subjects. Maximum SABD forces were be measured using a 6 degrees-of-freedom load cell (Model 45E15A; JR3, Woodland, CA), while study participants were strapped to a Biodex chair (Biodex Medical Systems, Shirley, NY), with the arm placed in a configuration of $75^{\circ} \mathrm{SABD}, 45^{\circ}$ shoulder flexion, $90^{\circ}$ elbow extension ( $180^{\circ}$ being full extension). Participants completed trials until they achieved three maximum voluntary SABD force values that were within $10 \%$ of each other.

Subsequently each subject performed a reaching task while generating a particular level of SABD, followed by a maximal attempt to open the hand at the end of each trial. Thumb and finger extension will be measured for three levels of maximum shoulder abduction (SABD) loads (table, 25\%, $50 \%)$.

A total of five $7 \mathrm{~mm}$ diameter infrared light emitting diodes (IRED) markers were placed at the tip of the thumb and fingers on each subject. Two motion capture systems (Optotrack Certus and Optotrack 3020) were used to track the markers' positions during the experiment. An ACT-3D robot (Fig.1) was used to generate the various SABD loads by providing forces perpendicular to the long axis of the arm. Subjects' wrists were tightly coupled to a forearm orthosis, 
and the thumb and fingers were relaxed in a grasping posture over a $60 \mathrm{~mm}$ diameter instrumented cylinder. At the end of the reach, subjects were instructed to open the hand as much as possible for maximal finger extension and thumb abduction.

TABLE I. SUBJECT INFORMATION

\begin{tabular}{|c|c|c|c|c|c|c|}
\hline Subject & Gender & $\begin{array}{c}\text { Affected } \\
\text { Side }\end{array}$ & Age & FM & Chedoke & Group \\
\hline $\mathbf{1}$ & M & R & $\mathbf{6 8}$ & $\mathbf{3 9}$ & $\mathbf{4}$ & Stroke \\
\hline $\mathbf{2}$ & M & R & $\mathbf{4 4}$ & $\mathbf{2 7}$ & $\mathbf{5}$ & Stroke \\
\hline 3 & M & L & $\mathbf{6 0}$ & $\mathbf{2 0}$ & $\mathbf{4}$ & Stroke \\
\hline 4 & M & L & $\mathbf{6 7}$ & $\mathbf{1 5}$ & $\mathbf{4}$ & Stroke \\
\hline $\mathbf{5}$ & F & R & $\mathbf{5 9}$ & & & Control \\
\hline 6 & M & R & $\mathbf{5 6}$ & & & Control \\
\hline 7 & M & R & $\mathbf{5 5}$ & & & Control \\
\hline
\end{tabular}

\section{Data Analysis}

The extension distance of thumb and four fingers were collected and quantified for three SABD load levels. This distance was normalized by the finger length for each individual. A three factor (group, SABD loading, finger) repeated measures analysis of variance (RMANOVA) was performed to determine the effect of group and SABD load level on extension distance. A paired t-test was performed as post-hoc within subject test, and a Bonferoni test was used as post-hoc text for between subject comparisons.

\section{RESULTS}

The RMANOVA resulted in a significance effect of group and level. Paired t-test demonstrated that thumb



extension was not significantly different for increased SABD load levels, but there was significantly decreased in extension for all fingers indicating an effect of the expression of the flexion synergy (Fig. 2 and Fig. 3).

Conversely, no significant differences between load levels were observed in age-matched able-bodied individuals.

\section{DISCUSSION}

The main finding of this preliminary study is the decrease in volitional finger extension as a function of SABD loading (table, $25 \%, 50 \%$ of the $\max$ ) in individuals with chronic stroke. This decrease was not observed in the able-bodied individuals. The expression of the flexion synergy thus reduces an individual's voluntary ability to control the hand

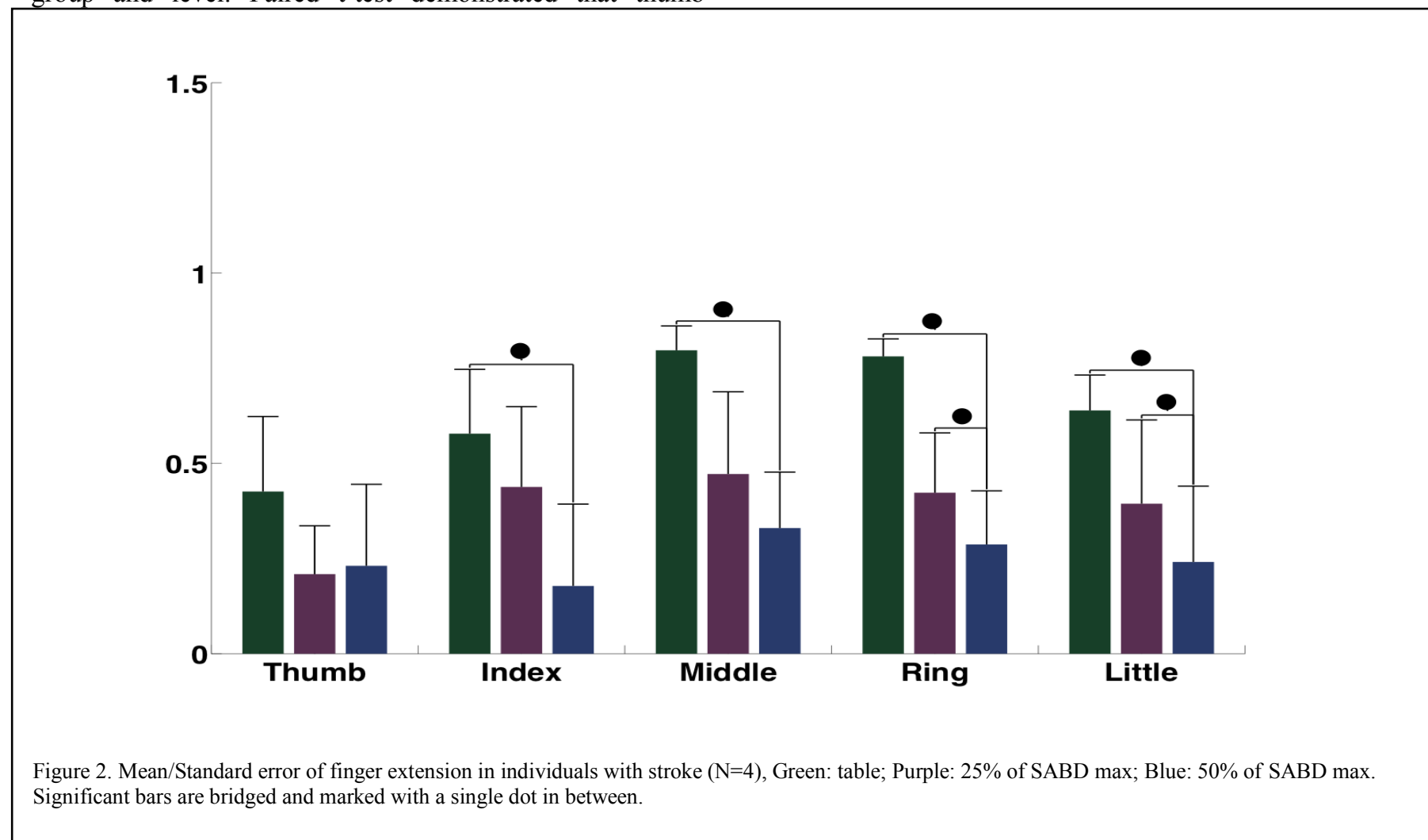




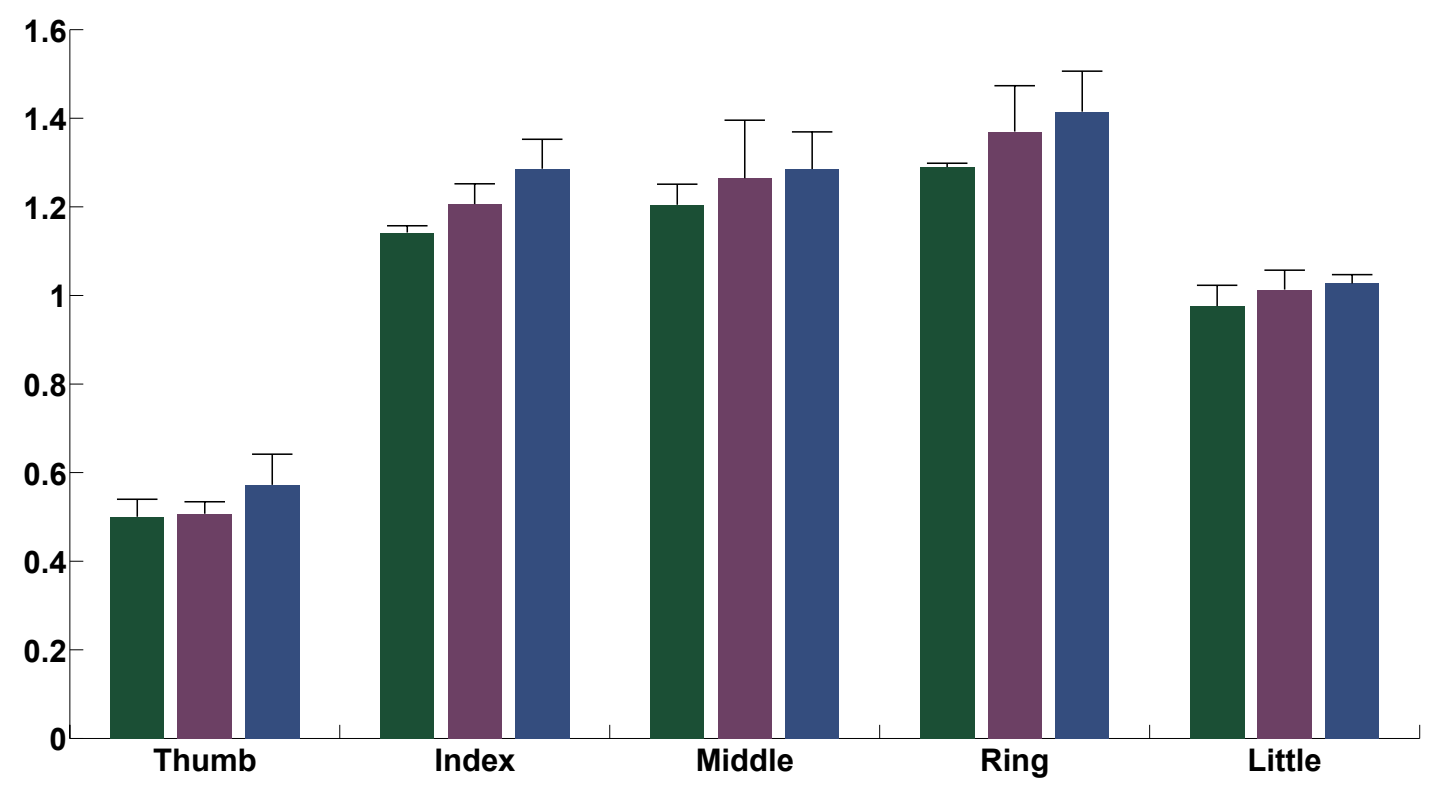

Figure 3. Mean/Standard error of finger extension in able-bodied individuals (N=3), Green: table; Purple: 25\% of SABD max; Blue: $50 \%$ of SABD max..

while abducting the shoulder and reaching with the paretic arm. Prior research indicated that due to flexion synergy, the activation of shoulder abductors significantly increased the activation of elbow flexors following stroke, and thus reducing the UE range of motion following stroke [8-10]. The impact of muscle synergies on hand function has received very little attention to date. Previous work from Miller and colleagues showed that shoulder abduction and reaching with the paretic arm significantly increases involuntary flexion forces at the wrist and fingers [5], pointing to the possible impact of the flexion synergy on post-stroke hand function.

Due to the stroke-induced losses of direct corticofugal projections, an increased reliance on an ipsilateral corticoreticulospinal pathway may result. The reticulospinal tract's diffuse projection onto various motoneuron pools that project to both proximal and distal muscles [11-14] explains the coactivation of muscles resulting in the flexion synergy. Even though projections from the cortico-reticulospinal pathway to wrist/finger muscles are found to be relatively weak in terms innervation ratio [15] compared to pyramidal tracts, this connectivity can be greatly utilized following losses of corticospinal projections. In short, an increase reliance on ispilateral cortico-reticulospinal projections explains the coactivation for shoulder abductor and elbow, wrist and finger flexors and the reduced ability to volitionally extend the fingers as SABD loading increases.

\section{ACKNOWLEDGMENT}

The authors would like to thank Justin Drogos and Carolina Carmona for assistance in recruiting and assessing study participants.

\section{REFERENCES}

[1] V. L. Roger, et al., "Heart disease and stroke statistics--2012 update: a report from the American Heart Association," Circulation, vol. 125, pp. e2-e220, Jan 32012.

[2] Broeks JG, et al., "The long-term outcome of arm function after stroke: results of a follow-up study," Disability and Rehabilitation, vol. 21 , pp. 357-64, 1999.

[3] D. Lloyd-Jones, et al., "Heart disease and stroke statistics--2010 update: a report from the American Heart Association," Circulation, vol. 121, pp. e46-e215, Feb 232010.

[4] L. P. Ince, Behavioral psychology in rehabilitation medicine : clinical applications. Baltimore: Williams \& Wilkins Co., 1980.

[5] L. C. Miller and J. P. Dewald, "Involuntary paretic wrist/finger flexion forces and EMG increase with shoulder abduction load in individuals with chronic stroke," Clinical neurophysiology : official journal of the International Federation of Clinical Neurophysiology, Feb 222012.

[6] L. C. Miller, et al., "A wrist and finger force sensor module for use during movements of the upper limb in chronic hemiparetic stroke," IEEE Trans Biomed Eng, vol. 56, pp. 2312-7, Sep 2009.

[7] S. Brunnstrom, Movement therapy in hemiplegia. New York: Harper and Row, 1970. [8] D. M. Corcos, et al., "Movement deficits caused by hyperexcitable stretch reflexes in spastic humans," Brain, vol. 109 ( Pt 5), pp. 1043-58, Oct 1986.

[8] J. Dewald and R. Beer, "Evidence for abnormal joint torque patterns in the paretic upper limb of subjects with hemiparesis.," Muscle \& Nerve, vol. 24, pp. 273-283, 2001.

[9] J. P. Dewald, et al., "Abnormal muscle coactivation patterns during isometric torque generation at the elbow and shoulder in hemiparetic subjects," Brain, vol. 118, pp. 495- 510, 1995.

[10] T. M. Sukal, et al., "Shoulder abduction-induced reductions in reaching work area following hemiparetic stroke: neuroscientific implications," Exp Brain Res, vol. 183, pp. 215-23, Nov 2007.

[11] A. G. Davidson and J. A. Buford, "Bilateral actions of the reticulospinal tract on arm and shoulder muscles in the monkey: stimulus triggered averaging," Experimental brain research. Experimentelle Hirnforschung. Experimentation cerebrale, vol. 173, pp. 25-39, Aug 2006.

[12] A. G. Davidson, et al., "Bilateral spike-triggered average effects in arm and shoulder muscles from the monkey pontomedullary reticular 
formation," The Journal of neuroscience : the official journal of the Society for Neuroscience, vol. 27, pp. 8053-8, Jul 252007.

[13] C. N. Riddle and S. N. Baker, "Convergence of pyramidal and medial brain stem descending pathways onto macaque cervical spinal interneurons," Journal of neurophysiology, vol. 103, pp. 2821-32, May 2010.

[14] C. N. Riddle, et al., "Direct and indirect connections with upper limb motoneurons from the primate reticulospinal tract," The Journal of neuroscience : the official journal of the Society for Neuroscience, vol. 29, pp. 4993-9, Apr 152009. 